\title{
VOC Emissions from Natural Upholstery Leathers
}

\author{
Petr Čech*, Jiří Stádník \\ Department of Furniture, Design and Habitation, Mendel University in Brno, Brno, Czech Republic
}

Received: 13 January 2020

Accepted: 24 March 2021

\begin{abstract}
This article addresses the issue of VOC emissions from upholstery materials (natural leather) used for the manufacturing of upholstered furniture. The main intention of this paper is to compare VOC emissions released from different types of natural upholstery leathers. This research has assessed the impact of various factors (e.g. the organic origin of leather, the type of tanning, the type of upholstery leather and the leather finish etc.) on the amount of VOC emissions from natural leathers used for the production of upholstered furniture. $1 \mathrm{~m}^{2}$ samples of natural leather were prepared for testing before being placed into a small test chamber $\left(1 \mathrm{~m}^{3}\right.$ volume). VOC emission measurements were taken in a small test chamber with predefined conditions (an ambient temperature of $23^{\circ} \mathrm{C}$ and $50 \%$ relative air humidity). With the use of sorbent Tenax TA were, the values of VOC emissions released by the test sample of different types of leather into desorption tubes was then recorded. VOC emissions were analysed in an Agilent GC $6890 \mathrm{~N}$ gas chromatograph using an Agilent 5973 Network mass spectrometer using cryofocusing, thermal desorption and a GC-MS data library (NIST 05). The analyses conducted provide qualitative and quantitative data regarding the concentration of selected VOC representatives measured in $\mu \mathrm{g} . \mathrm{m}^{-3}$. The quantitative difference in all emitted organic compounds is shown by the measured values of the TVOC parameter.
\end{abstract}

Keywords: VOC emissions, natural upholstery leathers, indoor air

\section{Introduction}

The indoor air pollution comes from sources inside the building, such as adhesives, carpeting, wooden or upholstery furniture and wood products or cleaning products, which may emit volatile organic compounds (VOCs) [1-4]. However, the outdoor air that enters the building can also be the main source of indoor air VOC pollution [5]. Therefore, if an appropriate ventilation

*e-mail: petr.cech@mendelu.cz, xstadnik@mendelu.cz rate with good quality outdoor air is applied to the dwelling, VOC concentration could be maintained in an acceptable level [6]. VOCs originate from both indoor and outdoor sources [7]; they are of particular concern due to their potential impact on human health. Formaldehyde and benzene [8], for example, are some of the most studied pollutants since they are classified in Group 1 of human carcinogens by the International Agency for Research on Cancer [9]. For many of these chemicals, the risk on human health and comfort is almost unknown and difficult to be predicted because of the lack of toxicological data. In the frame of the INDEX project [10] the existing knowledge worldwide 
has been assessed in terms of type and levels of chemicals in indoor air, as well as, the available toxicological information. It was concluded that VOCs such as benzene, formaldehyde, acetaldehyde, toluene and xylenes have to be considered as priority pollutants with respect to their health effects [11]. On the other hand, chemicals such as limonene and $\alpha$-pinene require further research with regard to human exposure or dose response and effects.

The main sources of aldehydes in homes include building materials, hardwood, plywood, laminate floorings, covering upholstery materials, adhesives, paints and varnishes and in some cases they are products of ozone- initiated reactions [12, 13]. For example, interior coatings can increase indoor air pollution due to VOC emissions [14]. Some of the major VOCs emitted from an oil-based varnish were ethylbenzene, m,pxylene, o-xylene and formaldehyde [15].

Soft furniture such as sofa, as an important part of civil furniture, has become one of the major sources of indoor air pollution. Due to the advantage of abundant resources and efficient function, leather has been the common raw and auxiliary materials of soft furniture [16].

Research study on the release of emissions Volatile Organic Compounds (VOCs) in natural leather samples by emission test chamber method found 21 kinds of VOCs detected from leather seat samples, including several types of aldehydes, ketones, aromatic hydrocarbons, alkanes, lipids etc [17]. The release trend of major volatile organic compounds in leather seats is consistent with the trend of total volatile organic compounds (TVOC). Both have shown a tendency of release rate from fast to slow and finally achieving a stable release [18].

One of the potentially important sources of organic emissions in leather seats comes from leather and other raw and auxiliary materials [19]. This may be because part of solvents, crosslinking agents and synthetic monomers remain in leather products after the pretreatment processing when a large number of additives (degreasing agent, crosslinking agent, etc.) and organic solvents (toluene, N,N-dimethyl formamide, etc.) are used [20]. Another experimental study reports the presence of specific chemical substances such as: 1,2-propandiol, 1-methoxy-2-propanol, N-ethyl-2pyrrolidone in natural upholstery leather samples [21].

\section{Material and Methods}

This research describes the VOC emissions emitted from covering materials used for upholstery furniture production, especially nature leathers. The main goal of this contribution is comparison of VOC emissions emitted from different types of nature upholstery leathers. This research judged the influence the impact of different factors (such as: animal origin, type of tanning, type of upholstery leathers etc.) on amount VOC emissions from natural leathers, that use for upholstery furniture production.

The select covering materials (natural leathers) were taken from the normal manufacturing process, wrapped in aluminium foils and delivered to the test laboratory. Natural leather samples were prepared from different type of materials in depending on these factors (animal species, type of tanning, type of upholstery leather).

The samples were formatted to the required sizes $(710 \times 710 \times 1 \mathrm{~mm})$ and then were divided into three groups depending on monitored factors. The first sample from tested samples was inserted into the test chamber (ČSN EN ISO 16000-9) immediately after unpacking from aluminium foil. The parameters of tested upholstery leather samples are shown in Tables 1 to 3 .

Then we started to collect VOC emission emitted by the tested sample into the desorption tubes on the sorbent Tenax TA (porous polymer based on 2,6-diphenyl oxide with a grain size of $0.18-0.25 \mathrm{~mm}$ ) [22].

The air sample in desorption tube with the captured sample of VOC emissions is then analysed in a gas chromatograph with a mass spectrometer and thermal desorption.

The conducted analyses provide qualitative and quantitative data on the concentrations of selected VOC and the total volatile organic compounds (TVOC) in $\mu \mathrm{g} . \mathrm{m}^{-3}$.

a) The influence of an organic type of leather (of animal origin) on VOC emissions

b) The influence of different types of tanning (chromeplated or tanned leather) on the amount of emission VOC emissions

c) Comparison of VOC emissions from different types of upholstery leathers

Table 1. The parameters of upholstery leather samples in dependence on animal origin.

\begin{tabular}{|c|c|c|c|c|c|c|c|c|}
\hline \multirow{2}{*}{ No. } & \multirow{2}{*}{ Type of tanning } & \multirow{2}{*}{$\begin{array}{c}\text { Country of } \\
\text { origin }\end{array}$} & \multicolumn{2}{|c|}{ Sizes of sample $(\mathrm{mm})$} & \multirow{2}{*}{ Animal origin } & \multirow{2}{*}{$\begin{array}{c}\text { Production } \\
\text { date }\end{array}$} & \multirow{2}{*}{ Colour } & \multirow{2}{*}{ Surface treatment } \\
\hline & & & Length & Width & & & & \\
\hline 1 & Chrome-plated & India & 710 & 710 & Buffalo & 9.1.2018 & Claret & Acrylic water-borne paint \\
\hline 2 & Chrome-plated & Brazil & 710 & 710 & Cow & 23.1.2018 & Anthracite & Acrylic water-borne paint \\
\hline 3 & Chrome-plated & India & 710 & 710 & Pig & 4.2.2018 & Grey & Acrylic water-borne paint \\
\hline
\end{tabular}


Table 2. The parameters of upholstery leather samples in dependence on different types of tanning.

\begin{tabular}{|c|c|c|c|c|c|c|c|c|}
\hline \multirow{2}{*}{ No. } & Type of tanning & \multirow{2}{*}{$\begin{array}{c}\text { Country of } \\
\text { origin }\end{array}$} & \multicolumn{2}{|c|}{$\begin{array}{c}\text { Sizes of sample } \\
(\mathrm{mm})\end{array}$} & \multirow{2}{*}{$\begin{array}{c}\text { Animal } \\
\text { origin }\end{array}$} & $\begin{array}{c}\text { Production } \\
\text { date }\end{array}$ & Colour & Surface treatment \\
\cline { 8 - 10 } & & Length & Width & Chrome-plated \\
1 & without preservatives & Brazil & 710 & 710 & Cow & 14.2 .2018 & Lrown & $\begin{array}{c}\text { Acrylic water-borne } \\
\text { paint }\end{array}$ \\
\hline 2 & $\begin{array}{c}\text { Chrome-plated } \\
\text { with preservatives }\end{array}$ & Brazil & 710 & 710 & Cow & 5.3 .2018 & Light brown & $\begin{array}{c}\text { Acrylic water-borne } \\
\text { paint }\end{array}$ \\
\hline 3 & $\begin{array}{c}\text { Tanned without } \\
\text { preservatives }\end{array}$ & Brazil & 710 & 710 & Cow & 12.3 .2018 & Brown & $\begin{array}{c}\text { Acrylic water-borne } \\
\text { paint }\end{array}$ \\
\hline 4 & $\begin{array}{c}\text { Tanned with } \\
\text { preservatives }\end{array}$ & Brazil & 710 & 710 & Cow & 15.3 .2018 & Light brown & $\begin{array}{c}\text { Acrylic water-borne } \\
\text { paint }\end{array}$ \\
\hline
\end{tabular}

Table 3. The parameters of upholstery leather samples in dependence on their comparison.

\begin{tabular}{|c|c|c|c|c|c|c|c|c|}
\hline \multirow{2}{*}{ No. } & \multirow{2}{*}{ Type of tanning } & \multirow{2}{*}{$\begin{array}{c}\text { Country of } \\
\text { origin }\end{array}$} & \multicolumn{2}{|c|}{ Sizes of sample $(\mathrm{mm})$} & \multirow{2}{*}{ Animal origin } & \multirow{2}{*}{$\begin{array}{l}\text { Production } \\
\text { date }\end{array}$} & \multirow{2}{*}{ Colour } & \multirow{2}{*}{ Surface treatment } \\
\hline & & & Length & Width & & & & \\
\hline 1 & Chrome-plated & India & 710 & 710 & Cow & 18.4.2018 & Brown & $\begin{array}{l}\text { Water-borne } \\
\text { two-tone effect }\end{array}$ \\
\hline 2 & Chrome-plated & Brazil & 710 & 710 & Cow & 25.4.2018 & Light green & $\begin{array}{l}\text { Water-borne } \\
\text { paint }\end{array}$ \\
\hline 3 & Chrome-plated & India & 710 & 710 & Cow & 10.5.2018 & Brown & $\begin{array}{l}\text { Pigmented with } \\
\text { two-tone effect }\end{array}$ \\
\hline 4 & Chrome-plated & Brazil & 710 & 710 & Cow & 16.5.2018 & Blue & $\begin{array}{c}\text { Water-borne } \\
\text { paint }\end{array}$ \\
\hline
\end{tabular}

\section{Methodology of Quantitative and Qualitative Determination of VOC Emissions}

Quantitative and qualitative determination of VOC emissions is performed on tested samples of covering materials especially on natural leathers. The effect of climatic conditions is also detected in the tested materials before the sampling of VOC emissions. The tested materials will be evaluated according to the time (age) from the date of production in the following time interval for 1, 3, 7, 28 and 120 days after the insertion of the samples into the VOC TEST 1000 small-space chamber.

The part of the measurement of VOC emissions is also the determination of total volatile organic compounds called TVOC (Total Volatile Organic Compounds. TVOC measurement is enabled by implementing an analytical and evaluation method within GC-MS.

\section{Air Samples}

In the present study, air samples from tested samples were collected onto Tenax TA adsorbent (sampling

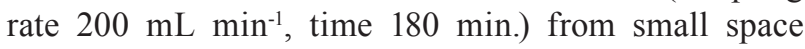
chamber (a volume $1 \mathrm{~m}^{3}$ ). Air samples were analysed with a gas chromatograph (HP 6890) equipped with a mass selective detector (MSD 5973) after thermal desorption at $250^{\circ} \mathrm{C}$ for $3 \mathrm{~min}$ (Scientific Instrument
Services TD4). The column was HP-5MS (column length $30 \mathrm{~m}$, i.d. $0,25 \mathrm{~mm}$, film thickness $1 \mu \mathrm{m}$ ), and the identification of the compounds was accomplished by retention times, standard compounds, and GC-MS data library (NIST 05).

The total VOC emission was first calculated by combining the peak areas of all identified compounds, after which the relative proportion of individual compounds from the total emission was calculated. The TVOC value is defined to be the integrated detector response value in toluene equivalents of compounds eluting between and including $\mathrm{C}_{6}$ to $\mathrm{C}_{16}$ as given in ISO 16000-6.

\section{Evaluation of VOC and VVOC}

The internal standard (D10-o-Xylen) method is used for the evaluation. Individual VOCs, VVOCs are evaluated by SIM recording. The results are evaluated in the ChemStation software environment after the completion of gas chromatograph analysis. The calibrated compounds are identified by the retention times, target and control ions in the SIM record and the software carries out the quantification of the analyte (ng.tube ${ }^{-1}$ ). The results are obtained from the report of the measured data of VOC and VVOC substances followed by the conversion to weight concentration $\left(\mu \mathrm{g} . \mathrm{m}^{-3}\right)$. The calculation is carried out according to Equation 1. 


$$
\rho_{x=\frac{m_{F}}{V}}
$$

In which: $\rho x$ is the mass concentration of the analyte in the air sample ( $\left.\mu \mathrm{g} \cdot \mathrm{m}^{-3}\right)$

$\mathrm{m}_{\mathrm{F}}$ is the amount of analyte captured on the tube (ng)

$\mathrm{V}$ is the collected air volume (liters)

\section{Results and Discussion}

This research study focused on the issue of VOC emissions from natural leathers used for the manufacture of upholstered furniture. Emissions from volatile organic compounds from different types of upholstery leather, in relation to the factors observed, were compared with the GC-MS analysis. VOC emissions from test samples were measured as a function of time (the time since insertion of the test sample into the test chamber) according the standard ČSN EN ISO 16000-9.
The experimental part of this study was divided into three parts:

a) To determine the influence of an organic type of leather (of animal origin) on VOC emissions.

b) To determine the influence of different types of tanning (chrome-plated or tanned leather) on the amount of VOC emissions.

c) To compare the VOC emissions from different types of upholstery leather.

The results of VOC emissions from natural upholstery leather samples in relation to animal species are shown in Tables 4-6. The test results show the measurement of more than 40 types of VOC released from the leather samples, including several types of aromatic substances, aldehydes, terpenes, and chemicals typical from natural leathers, such as: propylene glycol 1-methyl ether (synonym: 1-methoxy-2-propanol, CAS no. 107-98-2), Propylene glycol (synonym: 1,2 propanediol, CAS no.: 57-55-6); dipropylene glycol monomethyl ether (DPGMME, isomer-1, CAS no.13429-07-7) [23].

Table 4. VOC emission values from natural buffalo leather depending on the animal origin.

\begin{tabular}{|c|c|c|c|c|c|c|}
\hline & \multirow[t]{2}{*}{ VOCs } & \multicolumn{5}{|c|}{ Time dependence [days] } \\
\hline & & $1 \mathrm{D}$ & $3 \mathrm{D}$ & $7 \mathrm{D}$ & $28 \mathrm{D}$ & $120 \mathrm{D}$ \\
\hline No. & & $\begin{array}{l}\text { Average of } \\
\text { result } \pm \text { expanded } \\
\text { measurement } \\
\text { uncertainty }\end{array}$ & $\begin{array}{l}\text { Average of } \\
\text { result } \pm \text { expanded } \\
\text { measurement } \\
\text { uncertainty }\end{array}$ & $\begin{array}{c}\text { Average of } \\
\text { result } \pm \text { expanded } \\
\text { measurement } \\
\text { uncertainty }\end{array}$ & $\begin{array}{l}\text { Average of } \\
\text { result } \pm \text { expanded } \\
\text { measurement } \\
\text { uncertainty }\end{array}$ & $\begin{array}{l}\text { Average of } \\
\text { result } \pm \text { expanded } \\
\text { measurement } \\
\text { uncertainty }\end{array}$ \\
\hline & Unit & $\mu \mathrm{g} / \mathrm{m}^{3}$ & $\mu \mathrm{g} / \mathrm{m}^{3}$ & $\mu \mathrm{g} / \mathrm{m}^{3}$ & $\mu \mathrm{g} / \mathrm{m}^{3}$ & $\mu \mathrm{g} / \mathrm{m}^{3}$ \\
\hline 1. & Formaldehyde & $<3$ & $<3$ & $<3$ & $<3$ & $<3$ \\
\hline 2. & 1,3-Butadiene & $<0.3$ & $<0.3$ & $<0.3$ & $<0.3$ & $<0.3$ \\
\hline 3. & Acetone & $<0.1$ & $<0.1$ & $<0.1$ & $<0.1$ & $<0.1$ \\
\hline 4. & Butanal & $<0.1$ & $<0.1$ & $<0.1$ & $<0.1$ & $<0.1$ \\
\hline 5. & Ethyl acetate & $<0.1$ & $<0.1$ & $<0.1$ & $<0.1$ & $<0.1$ \\
\hline 6. & Crotonaldehyde & $(0.2 \pm 0.06)$ & $(0.4 \pm 0.1)$ & $(0.3 \pm 0.1)$ & $<0.1$ & $<0.1$ \\
\hline 7. & Benzene & $<0.1$ & $(0.2 \pm 0.06)$ & $<0.1$ & $<0.1$ & $(0.1 \pm 0.03)$ \\
\hline 8. & 1-Methoxy, 2-propanol & $(30.3 \pm 9.1)$ & $(15.1 \pm 4.5)$ & $(10.9 \pm 3.3)$ & $(0.9 \pm 0.3)$ & $(0.2 \pm 0.06)$ \\
\hline 9. & Pentanal & $(0.3 \pm 0.1)$ & $(0.3 \pm 0.1)$ & $(0.5 \pm 0.2)$ & $<0.1$ & $<0.1$ \\
\hline 10. & Trichlorethylene & $<0.1$ & $<0.1$ & $<0.1$ & $<0.1$ & $<0.1$ \\
\hline 11. & Toluene & $(0.4 \pm 0.1)$ & $(1.9 \pm 0.6)$ & $(3.9 \pm 1.2)$ & $<0.1$ & $<0.1$ \\
\hline 12. & Hexanal & $(2.7 \pm 0.8)$ & $(1.9 \pm 0.6)$ & $(0.9 \pm 0.3)$ & $(0.2 \pm 0.06)$ & $(0.1 \pm 0.03)$ \\
\hline 13. & Tetrachlorethylene & $<0.1$ & $(0.1 \pm 0.03)$ & $(0.1 \pm 0.03)$ & $(0.1 \pm 0.03)$ & $(0.1 \pm 0.03)$ \\
\hline 14. & n-Butyl acetate & $(0.2 \pm 0.06)$ & $(0.4 \pm 0.1)$ & $(0.2 \pm 0.06)$ & $(4.4 \pm 1.3)$ & $(0.1 \pm 0.03)$ \\
\hline 15. & Furfural & $(0.2 \pm 0.06)$ & $(0.1 \pm 0.03)$ & $(0.1 \pm 0.03)$ & $<0.1$ & $(0.1 \pm 0.03)$ \\
\hline 16. & Ethylbenzene & $(0.1 \pm 0.03)$ & $(0.5 \pm 0.2)$ & $(0.4 \pm 0.1)$ & $<0.1$ & $(2 \pm 0.6)$ \\
\hline 17. & m,p-Xylene & $(0.2 \pm 0.06)$ & $(0.7 \pm 0.2)$ & $(0.9 \pm 0.3)$ & $<0.1$ & $(6.1 \pm 1.8)$ \\
\hline 18. & Styrene & $<0.1$ & $(0.1 \pm 0.03)$ & $(0.1 \pm 0.03)$ & $<0.1$ & $<0.1$ \\
\hline 19. & 1,2 propandiol & $(7.8 \pm 2.3)$ & $(6.3 \pm 1.9)$ & $(0.3 \pm 0.1)$ & $(0.1 \pm 0.03)$ & $(0.1 \pm 0.03)$ \\
\hline
\end{tabular}


Table 4. Continued.

\begin{tabular}{|c|c|c|c|c|c|c|}
\hline 20. & Cyclohexanone & $<0.1$ & $<0.1$ & $<0.1$ & $<0.1$ & $<0.1$ \\
\hline 21. & o-Xylene & $(0.1 \pm 0.03)$ & $(0.3 \pm 0.1)$ & $(0.2 \pm 0.06)$ & $(0.1 \pm 0.03)$ & $(1.5 \pm 0.5)$ \\
\hline 22. & Heptanal & $(1.1 \pm 0.3)$ & $(0.4 \pm 0.1)$ & $(0.2 \pm 0.06)$ & $(0.1 \pm 0.03)$ & $<0.1$ \\
\hline 23. & Butoxy-Ethanol & $(53.5 \pm 16.1)$ & $(28.4 \pm 8.5)$ & $(7.8 \pm 2.3)$ & $(1.4 \pm 0.4)$ & $(0.2 \pm 0.06)$ \\
\hline 24. & $\alpha$-Pinene & $<0.1$ & $(0.2 \pm 0.06)$ & $(1 \pm 0.3)$ & $<0.1$ & $<0.1$ \\
\hline 25. & Camphene & $<0.1$ & $<0.1$ & $(0.1 \pm 0.03)$ & $<0.1$ & $<0.1$ \\
\hline 26. & Benzaldehyde & $(1.1 \pm 0.3)$ & $(0.4 \pm 0.1)$ & $(0.2 \pm 0.06)$ & $(0.2 \pm 0.06)$ & $(0.1 \pm 0.03)$ \\
\hline 27. & 3-Ethyl-Toluene & $(0.2 \pm 0.06)$ & $(0.1 \pm 0.03)$ & $(0.1 \pm 0.03)$ & $(0.1 \pm 0.03)$ & $<0.1$ \\
\hline 28. & 4-Ethyl-Toluene & $(0.3 \pm 0.1)$ & $<0.1$ & $(0.1 \pm 0.03)$ & $(0.1 \pm 0.03)$ & $<0.1$ \\
\hline 29. & 1.3.5-Trimethyl-Bnezene & $(0.1 \pm 0.03)$ & $<0.1$ & $<0.1$ & $(0.1 \pm 0.03)$ & $<0.1$ \\
\hline 30. & Phenol & $(1 \pm 0.3)$ & $(0.9 \pm 0.3)$ & $(0.8 \pm 0.2)$ & $(0.7 \pm 0.2)$ & $(0.5 \pm 0.2)$ \\
\hline 31. & $\beta$-Pinene & $<0.1$ & $(0.1 \pm 0.03)$ & $(0.1 \pm 0.03)$ & $<0.1$ & $<0.1$ \\
\hline 32. & 2-Ethyl_Toluene & $<0.1$ & $<0.1$ & $<0.1$ & $<0.1$ & $<0.1$ \\
\hline 33. & Myrcene & $<0.1$ & $<0.1$ & $(0.1 \pm 0.03)$ & $(0.2 \pm 0.06)$ & $<0.1$ \\
\hline 34. & 1.2.4-Trimethyl-Benzene & $<0.1$ & $<0.1$ & $<0.1$ & $(0.1 \pm 0.03)$ & $<0.1$ \\
\hline 35. & Octanal & $(2.7 \pm 0.8)$ & $(0.8 \pm 0.2)$ & $(0.7 \pm 0.2)$ & $<0.1$ & $<0.1$ \\
\hline 36. & $\alpha$-Phelandren & $<0.1$ & $<0.1$ & $<0.1$ & $<0.1$ & $<0.1$ \\
\hline 37. & 3- $\delta$-Carene & $<0.1$ & $(0.3 \pm 0.1)$ & $(0.3 \pm 0.1)$ & $<0.1$ & $<0.1$ \\
\hline 38. & 1.4-Dichlorbenzene & $<0.1$ & $<0.1$ & $<0.1$ & $<0.1$ & $<0.1$ \\
\hline 39. & DPGMME. isomer 1 & $(0.3 \pm 0.1)$ & $(0.3 \pm 0.1)$ & $(0.2 \pm 0.06)$ & $(0.2 \pm 0.06)$ & $(0.1 \pm 0.03)$ \\
\hline 40. & 1.2.3-Trimethyl-Benzene & $<0.1$ & $(0.1 \pm 0.03)$ & $(0.1 \pm 0.03)$ & $(0.1 \pm 0.03)$ & $<0.1$ \\
\hline 41. & Limonene & $(0.1 \pm 0.03)$ & $(0.2 \pm 0.06)$ & $(0.1 \pm 0.03)$ & $<0.1$ & $<0.1$ \\
\hline 42. & $\gamma$-Terpinene & $<0.1$ & $<0.1$ & $<0.1$ & $<0.1$ & $<0.1$ \\
\hline 43. & Nonanal & $(18.1 \pm 5.4)$ & $(8.2 \pm 2.5)$ & $(3.8 \pm 1.1)$ & $(2.5 \pm 0.8)$ & $<0.1$ \\
\hline 44. & Decanal & $<1.1$ & $<1.1$ & $<1.1$ & $<1.1$ & $<1.1$ \\
\hline 45. & Bornyl acetate & $<0.1$ & $<0.1$ & $<0.1$ & $<0.1$ & $<0.1$ \\
\hline 46. & $\mathrm{TVOC}_{\mathrm{MS}}$ & $(548 \pm 164)$ & $(336 \pm 101)$ & $(272 \pm 82)$ & $(158 \pm 47)$ & $(118 \pm 35)$ \\
\hline
\end{tabular}

Table 5. VOC emission values from natural cow leather depending on the animal origin.

\begin{tabular}{|c|c|c|c|c|c|c|}
\hline & \multirow[t]{2}{*}{ VOCs } & \multicolumn{5}{|c|}{ Time dependence [days] } \\
\hline & & $1 \mathrm{D}$ & $3 \mathrm{D}$ & $7 \mathrm{D}$ & $28 \mathrm{D}$ & $120 \mathrm{D}$ \\
\hline No. & & $\begin{array}{l}\text { Average of } \\
\text { result } \pm \text { expanded } \\
\text { measurement } \\
\text { uncertainty }\end{array}$ & $\begin{array}{l}\text { Average of } \\
\text { result } \pm \text { expanded } \\
\text { measurement } \\
\text { uncertainty }\end{array}$ & $\begin{array}{l}\text { Average of } \\
\text { result } \pm \text { expanded } \\
\text { measurement } \\
\text { uncertainty }\end{array}$ & $\begin{array}{c}\text { Average of } \\
\text { result } \pm \text { expanded } \\
\text { measurement } \\
\text { uncertainty }\end{array}$ & $\begin{array}{l}\text { Average of } \\
\text { result } \pm \text { expanded } \\
\text { measurement } \\
\text { uncertainty }\end{array}$ \\
\hline & Unit & $\mu \mathrm{g} / \mathrm{m}^{3}$ & $\mu \mathrm{g} / \mathrm{m}^{3}$ & $\mu \mathrm{g} / \mathrm{m}^{3}$ & $\mu \mathrm{g} / \mathrm{m}^{3}$ & $\mu \mathrm{g} / \mathrm{m}^{3}$ \\
\hline 1. & Formaldehyde & $<3$ & $<3$ & $<3$ & $<3$ & $<3$ \\
\hline 2. & 1,3-Butadiene & $<0.3$ & $<0.3$ & $<0.3$ & $<0.3$ & $<0.3$ \\
\hline 3. & Acetone & $<0.1$ & $<0.1$ & $<0.1$ & $<0.1$ & $<0.1$ \\
\hline 4. & Butanal & $<0.1$ & $<0.1$ & $<0.1$ & $<0.1$ & $<0.1$ \\
\hline 5. & Ethyl acetate & $<0.1$ & $<0.1$ & $<0.1$ & $<0.1$ & $<0.1$ \\
\hline 6. & Crotonaldehyde & $<0.1$ & $(0.1 \pm 0.03)$ & $<0.1$ & $(0.1 \pm 0.03)$ & $<0.1$ \\
\hline
\end{tabular}


Table 5. Continued.

\begin{tabular}{|c|c|c|c|c|c|c|}
\hline 7. & Benzene & $<0.1$ & $<0.1$ & $<0.1$ & $<0.1$ & $(0.1 \pm 0.03)$ \\
\hline 8. & 1-Methoxy, 2-propanol & $(20.0 \pm 6)$ & $(12.3 \pm 3.7)$ & $(3.6 \pm 1.1)$ & $(0.1 \pm 0.03)$ & $(0.1 \pm 0.03)$ \\
\hline 9. & Pentanal & $(0.1 \pm 0.03)$ & $(0.1 \pm 0.03)$ & $(0.1 \pm 0.03)$ & $(0.4 \pm 0.1)$ & $<0.1$ \\
\hline 10. & Trichlorethylene & $<0.1$ & $<0.1$ & $<0.1$ & $<0.1$ & $<0.1$ \\
\hline 11. & Toluene & $<0.1$ & $(0.1 \pm 0.03)$ & $<0.1$ & $(2.5 \pm 0.8)$ & $(0.1 \pm 0.03)$ \\
\hline 12. & Hexanal & $(2.8 \pm 0.8)$ & $(2.0 \pm 0.6)$ & $(1.6 \pm 0.5)$ & $(1.3 \pm 0.4)$ & $<0.1$ \\
\hline 13. & Tetrachlorethylene & $<0.1$ & $<0.1$ & $<0.1$ & $<0.1$ & $<0.1$ \\
\hline 14. & n-Butyl acetate & $<0.1$ & $<0.1$ & $<0.1$ & $(0.1 \pm 0.03)$ & $(0.1 \pm 0.03)$ \\
\hline 15. & Furfural & $(0.2 \pm 0.06)$ & $(0.1 \pm 0.03)$ & $(0.1 \pm 0.03)$ & $(0.1 \pm 0.03)$ & $<0.1$ \\
\hline 16. & Ethylbenzene & $<0.1$ & $(0.1 \pm 0.03)$ & $<0.1$ & $(0.5 \pm 0.2)$ & $(0.1 \pm 0.03)$ \\
\hline 17. & m,p-Xylene & $<0.1$ & $(0.1 \pm 0.03)$ & $(4.7 \pm 1.4)$ & $(0.1 \pm 0.03)$ & $<0.1$ \\
\hline 18. & Styrene & $<0.1$ & $(0.1 \pm 0.03)$ & $<0.1$ & $(0.3 \pm 0.1)$ & $<0.1$ \\
\hline 19. & 1,2 propandiol & $(4.0 \pm 1.2)$ & $(3.2 \pm 1)$ & $(0.3 \pm 0.1)$ & $(0.2 \pm 0.06)$ & $(0.1 \pm 0.03)$ \\
\hline 20. & Cyclohexanone & $<0.1$ & $<0.1$ & $<0.1$ & $<0.1$ & $<0.1$ \\
\hline 21. & o-Xylene & $<0.1$ & $<0.1$ & $<0.1$ & $(1.2 \pm 0.4)$ & $(0.1 \pm 0.03)$ \\
\hline 22. & Heptanal & $(0.1 \pm 0.03)$ & $(0.1 \pm 0.03)$ & $<0.1$ & $<0.1$ & $<0.1$ \\
\hline 23. & Butoxy-Ethanol & $(44.3 \pm 13.3)$ & $(22.4 \pm 6.7)$ & $(6.3 \pm 1.9)$ & $(0.2 \pm 0.06)$ & $(0.1 \pm 0.03)$ \\
\hline 24. & $\alpha$-Pinene & $<0.1$ & $<0.1$ & $(0.1 \pm 0.03)$ & $(1 \pm 0.3)$ & $<0.1$ \\
\hline 25. & Camphene & $<0.1$ & $<0.1$ & $<0.1$ & $(0.2 \pm 0.06)$ & $<0.1$ \\
\hline 26. & Benzaldehyde & $(2.1 \pm 0.6)$ & $(0.6 \pm 0.2)$ & $(0.1 \pm 0.03)$ & $(0.2 \pm 0.06)$ & $<0.1$ \\
\hline 27. & 3-Ethyl-Toluene & $(0.4 \pm 0.1)$ & $(0.1 \pm 0.03)$ & $<0.1$ & $<0.1$ & $<0.1$ \\
\hline 28. & 4-Ethyl-Toluene & $<0.1$ & $<0.1$ & $<0.1$ & $<0.1$ & $<0.1$ \\
\hline 29. & 1,3,5-Trimethyl-Bnezene & $<0.1$ & $<0.1$ & $<0.1$ & $(0.1 \pm 0.03)$ & $<0.1$ \\
\hline 30. & Phenol & $(0.4 \pm 0.1)$ & $(0.4 \pm 0.1)$ & $(0.4 \pm 0.1)$ & $(0.2 \pm 0.06)$ & $(0.4 \pm 0.1)$ \\
\hline 31. & $\beta$-Pinene & $<0.1$ & $<0.1$ & $<0.1$ & $<0.1$ & $<0.1$ \\
\hline 32. & 2-Ethyl_Toluene & $<0.1$ & $<0.1$ & $<0.1$ & $(0.1 \pm 0.03)$ & $<0.1$ \\
\hline 33. & Myrcene & $<0.1$ & $<0.1$ & $<0.1$ & $<0.1$ & $<0.1$ \\
\hline 34. & 1,2,4-Trimethyl-Benzene & $<0.1$ & $(0.1 \pm 0.03)$ & $<0.1$ & $(0.1 \pm 0.03)$ & $<0.1$ \\
\hline 35. & Octanal & $<0.1$ & $(0.2 \pm 0.06)$ & $(0.1 \pm 0.03)$ & $<0.1$ & $<0.1$ \\
\hline 36. & $\alpha$-Phelandren & $<0.1$ & $<0.1$ & $<0.1$ & $<0.1$ & $<0.1$ \\
\hline 37. & 3 - $\delta$-Carene & $<0.1$ & $(0.1 \pm 0.03)$ & $(0.1 \pm 0.03)$ & $(0.5 \pm 0.2)$ & $<0.1$ \\
\hline 38. & 1,4-Dichlorbenzene & $<0.1$ & $<0.1$ & $<0.1$ & $<0.1$ & $<0.1$ \\
\hline 39. & DPGMME, isomer 1 & $(0.2 \pm 0.06)$ & $(0.2 \pm 0.06)$ & $(0.2 \pm 0.06)$ & $(0.1 \pm 0.03)$ & $(0.1 \pm 0.03)$ \\
\hline 40. & 1,2,3-Trimethyl-Benzene & $<0.1$ & $<0.1$ & $<0.1$ & $<0.1$ & $<0.1$ \\
\hline 41. & Limonene & $<0.1$ & $(2.9 \pm 0.9)$ & $(0.1 \pm 0.03)$ & $(0.3 \pm 0.1)$ & $(0.1 \pm 0.03)$ \\
\hline 42. & $\gamma$-Terpinene & $<0.1$ & $<0.1$ & $<0.1$ & $<0.1$ & $<0.1$ \\
\hline 43. & Nonanal & $(1.7 \pm 0.5)$ & $(0.7 \pm 0.2)$ & $(0.3 \pm 0.1)$ & $<0.1$ & $<0.1$ \\
\hline 44. & Decanal & $<1.1$ & $<1.1$ & $<1.1$ & $<1.1$ & $<1.1$ \\
\hline 45. & Bornyl acetate & $<0.1$ & $<0.1$ & $<0.1$ & $<0.1$ & $<0.1$ \\
\hline 46. & TVOC $_{\mathrm{MS}}$ & $(422 \pm 127)$ & $(272 \pm 82)$ & $(197 \pm 59)$ & $(128 \pm 38)$ & $(91 \pm 27)$ \\
\hline
\end{tabular}


Table 6. VOC emission values from natural pig leather depending on the animal origin.

\begin{tabular}{|c|c|c|c|c|c|c|}
\hline & \multirow[t]{2}{*}{ VOCs } & \multicolumn{5}{|c|}{ Time dependence [days] } \\
\hline & & $1 \mathrm{D}$ & $3 \mathrm{D}$ & $7 \mathrm{D}$ & $28 \mathrm{D}$ & $120 \mathrm{D}$ \\
\hline No. & & $\begin{array}{l}\text { Average of } \\
\text { result } \pm \text { expanded } \\
\text { measurement } \\
\text { uncertainty }\end{array}$ & $\begin{array}{c}\text { Average of } \\
\text { result } \pm \text { expanded } \\
\text { measurement } \\
\text { uncertainty }\end{array}$ & $\begin{array}{c}\text { Average of } \\
\text { result } \pm \text { expanded } \\
\text { measurement } \\
\text { uncertainty }\end{array}$ & $\begin{array}{c}\text { Average of } \\
\text { result } \pm \text { expanded } \\
\text { measurement } \\
\text { uncertainty }\end{array}$ & $\begin{array}{c}\text { Average of } \\
\text { result } \pm \text { expanded } \\
\text { measurement } \\
\text { uncertainty }\end{array}$ \\
\hline & Unit & $\mu \mathrm{g} / \mathrm{m}^{3}$ & $\mu \mathrm{g} / \mathrm{m}^{3}$ & $\mu \mathrm{g} / \mathrm{m}^{3}$ & $\mu \mathrm{g} / \mathrm{m}^{3}$ & $\mu \mathrm{g} / \mathrm{m}^{3}$ \\
\hline 1. & Formaldehyde & $<3$ & $<3$ & $<3$ & $<3$ & $<3$ \\
\hline 2. & 1,3-Butadiene & $<0.3$ & $<0.3$ & $<0.3$ & $<0.3$ & $<0.3$ \\
\hline 3. & Acetone & $<0.1$ & $<0.1$ & $<0.1$ & $<0.1$ & $<0.1$ \\
\hline 4. & Butanal & $<0.1$ & $<0.1$ & $<0.1$ & $<0.1$ & $<0.1$ \\
\hline 5. & Ethyl acetate & $<0.1$ & $<0.1$ & $<0.1$ & $<0.1$ & $<0.1$ \\
\hline 6. & Crotonaldehyde & $<0.1$ & $(0.1 \pm 0.03)$ & $<0.1$ & $(0.1 \pm 0.03)$ & $<0.1$ \\
\hline 7. & Benzene & $<0.1$ & $<0.1$ & $<0.1$ & $<0.1$ & $<0.1$ \\
\hline 8. & 1-Methoxy, 2-propanol & $(16.5 \pm 5)$ & $(1.9 \pm 0.6)$ & $(0.2 \pm 0.06)$ & $(0.1 \pm 0.03)$ & $<0.1$ \\
\hline 9. & Pentanal & $<0.1$ & $<0.1$ & $<0.1$ & $(0.3 \pm 0.1)$ & $<0.1$ \\
\hline 10. & Trichlorethylene & $<0.1$ & $<0.1$ & $<0.1$ & $<0.1$ & $<0.1$ \\
\hline 11. & Toluene & $(12.4 \pm 3.7)$ & $(2.8 \pm 0.8)$ & $(2.0 \pm 0.6)$ & $(1.8 \pm 0.5)$ & $(0.4 \pm 0.1)$ \\
\hline 12. & Hexanal & $(2.2 \pm 0.7)$ & $(1.5 \pm 0.5)$ & $(1.2 \pm 0.4)$ & $(0.9 \pm 0.3)$ & $<0.1$ \\
\hline 13. & Tetrachlorethylene & $<0.1$ & $<0.1$ & $<0.1$ & $<0.1$ & $<0.1$ \\
\hline 14. & n-Butyl acetate & $<0.1$ & $<0.1$ & $<0.1$ & $<0.1$ & $(3 \pm 0.9)$ \\
\hline 15. & Furfural & $(0.2 \pm 0.06)$ & $<0.1$ & $<0.1$ & $(0.1 \pm 0.03)$ & $<0.1$ \\
\hline 16. & Ethylbenzene & $<0.1$ & $<0.1$ & $<0.1$ & $(0.3 \pm 0.1)$ & $(1.3 \pm 0.4)$ \\
\hline 17. & $\mathrm{~m}, \mathrm{p}$-Xylene & $(4.0 \pm 1.2)$ & $<0.1$ & $<0.1$ & $(0.7 \pm 0.2)$ & $(0.3 \pm 0.1)$ \\
\hline 18. & Styrene & $<0.1$ & $(0.1 \pm 0.03)$ & $<0.1$ & $(0.2 \pm 0.06)$ & $<0.1$ \\
\hline 19. & 1,2 propandiol & $(2.3 \pm 0.7)$ & $(1.8 \pm 0.5)$ & $(1.1 \pm 0.3)$ & $(0.4 \pm 0.1)$ & $(0.3 \pm 0.1)$ \\
\hline 20. & Cyclohexanone & $<0.1$ & $<0.1$ & $<0.1$ & $<0.1$ & $<0.1$ \\
\hline 21. & o-Xylene & $(1 \pm 0.3)$ & $(0.1 \pm 0.03)$ & $(0.1 \pm 0.03)$ & $(0.1 \pm 0.03)$ & $<0.1$ \\
\hline 22. & Heptanal & $<0.1$ & $<0.1$ & $<0.1$ & $<0.1$ & $<0.1$ \\
\hline 23. & Butoxy-Ethanol & $(36.6 \pm 11)$ & $(18.5 \pm 5.6)$ & $(6.1 \pm 1.8)$ & $(0.1 \pm 0.03)$ & $(1 \pm 0.3)$ \\
\hline 24. & $\alpha$-Pinene & $<0.1$ & $<0.1$ & $<0.1$ & $(0.8 \pm 0.2)$ & $<0.1$ \\
\hline 25. & Camphene & $<0.1$ & $<0.1$ & $<0.1$ & $(0.1 \pm 0.03)$ & $<0.1$ \\
\hline 26. & Benzaldehyde & $(1.7 \pm 0.5)$ & $(0.4 \pm 0.1)$ & $<0.1$ & $(0.1 \pm 0.03)$ & $<0.1$ \\
\hline 27. & 3-Ethyl-Toluene & $(0.2 \pm 0.06)$ & $<0.1$ & $<0.1$ & $<0.1$ & $<0.1$ \\
\hline 28. & 4-Ethyl-Toluene & $<0.1$ & $<0.1$ & $<0.1$ & $<0.1$ & $<0.1$ \\
\hline 29. & 1,3,5-Trimethyl-Benzene & $<0.1$ & $<0.1$ & $<0.1$ & $<0.1$ & $<0.1$ \\
\hline 30. & Phenol & $(0.3 \pm 0.1)$ & $(0.3 \pm 0.1)$ & $(0.3 \pm 0.1)$ & $(0.1 \pm 0.03)$ & $(0.4 \pm 0.1)$ \\
\hline 31. & $\beta$-Pinene & $<0.1$ & $<0.1$ & $<0.1$ & $<0.1$ & $<0.1$ \\
\hline 32. & 2-Ethyl_Toluene & $<0.1$ & $<0.1$ & $<0.1$ & $<0.1$ & $<0.1$ \\
\hline 33. & Myrcene & $<0.1$ & $<0.1$ & $<0.1$ & $<0.1$ & $<0.1$ \\
\hline 34. & 1,2,4-Trimethyl-Benzene & $<0.1$ & $(0.1 \pm 0.03)$ & $<0.1$ & $(0.1 \pm 0.03)$ & $<0.1$ \\
\hline 35. & Octanal & $<0.1$ & $(0.1 \pm 0.03)$ & $<0.1$ & $<0.1$ & $<0.1$ \\
\hline 36. & $\alpha$-Phelandren & $<0.1$ & $<0.1$ & $<0.1$ & $<0.1$ & $<0.1$ \\
\hline
\end{tabular}


Table 6. Continued.

\begin{tabular}{|c|c|c|c|c|c|c|}
\hline 37. & 3- $\delta$-Carene & $<0.1$ & $<0.1$ & $<0.1$ & $(0.4 \pm 0.1)$ & $<0.1$ \\
\hline 38. & 1,4-Dichlorbenzene & $<0.1$ & $<0.1$ & $<0.1$ & $<0.1$ & $<0.1$ \\
\hline 39. & DPGMME, isomer 1 & $(0.2 \pm 0.06)$ & $(0.1 \pm 0.03)$ & $(0.1 \pm 0.03)$ & $(0.1 \pm 0.03)$ & $(0.1 \pm 0.03)$ \\
\hline 40. & $1,2,3$-Trimethyl-Benzene & $<0.1$ & $<0.1$ & $<0.1$ & $<0.1$ & $<0.1$ \\
\hline 41. & Limonene & $<0.1$ & $(0.4 \pm 0.1)$ & $(0.1 \pm 0.03)$ & $(0.2 \pm 0.06)$ & $(0.1 \pm 0.03)$ \\
\hline 42. & $\gamma$-Terpinene & $<0.1$ & $<0.1$ & $<0.1$ & $<0.1$ & $<0.1$ \\
\hline 43. & Nonanal & $(1.2 \pm 0.4)$ & $(0.4 \pm 0.1)$ & $(0.1 \pm 0.03)$ & $<0.1$ & $<0.1$ \\
\hline 44. & Decanal & $<1.1$ & $<1.1$ & $<1.1$ & $<1.1$ & $<1.1$ \\
\hline 45. & Bornyl acetate & $<0.1$ & $<0.1$ & $<0.1$ & $<0.1$ & $<0.1$ \\
\hline 46. & TVOC $_{\text {MS }}$ & $(346 \pm 104)$ & $(223 \pm 67)$ & $(160 \pm 48)$ & $(103 \pm 31)$ & $(78 \pm 23)$ \\
\hline
\end{tabular}

a) The influence of an organic type of leather (of animal origin) on the amount of VOC emissions.

b) The influence of different types of tanning (chromeplated or tanned leather) on the amount of emission VOC emissions.

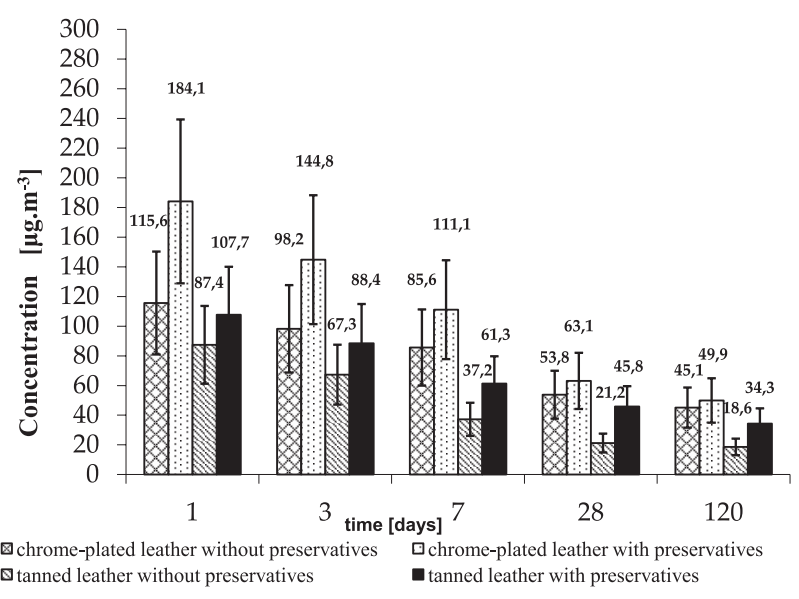

Fig. 1. 1-Methoxy, 2-propanol from natural leathers based on different type of tanning.

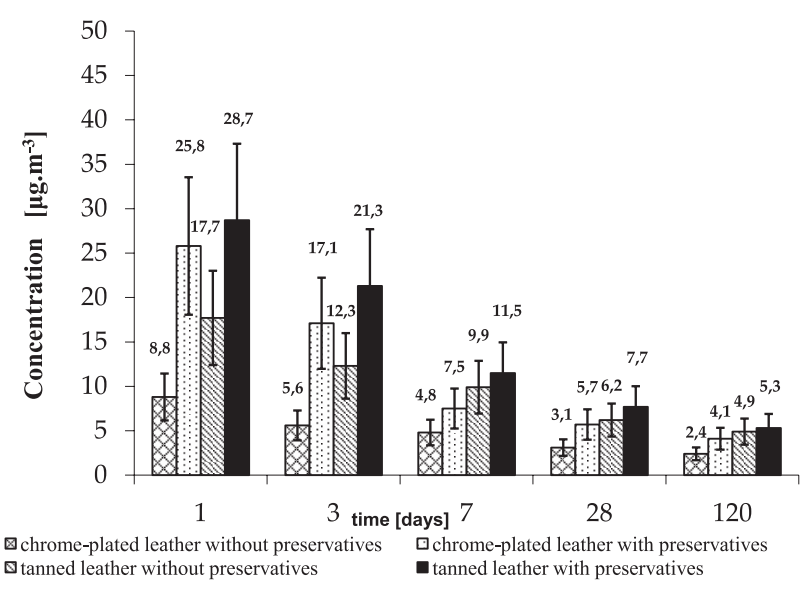

Fig. 2. Butoxy-Ethanol from natural leathers based on different types of tanning.
Fig. 1 shows the influence of different types of tanning from natural leathers on the quantity of emissions VOC, especially concentration of 1-Methoxy, 2-propanol. The highest concentration of 1-Methoxy, 2-propanol was found from chrome-plated leather with preservatives at measurements after 1 day in amount over $184 \mu \mathrm{g} . \mathrm{m}^{-3}$.

Fig. 2 presents the different types of tanning from natural leathers on the quantity of emissions VOC, especially the concentration of Butoxy Ethanol. The highest concentration of Butoxy-Ethanol was found from tanned leather with preservatives at measurements after 1 day.

Fig. 3 presents data of TVOC (Total Volatile Organic Compounds) from different types of tanning from natural leathers. The highest amount of TVOC emitted by tanned leather with preservatives, especially at measurements after 72 hours.

a) Comparison of VOC emissions from different types of upholstery leathers.

Fig. 4 there presents different types of upholstery leathers on content of emissions VOC, especially of emissions of 1_Methoxy_2_Propanol. The highest

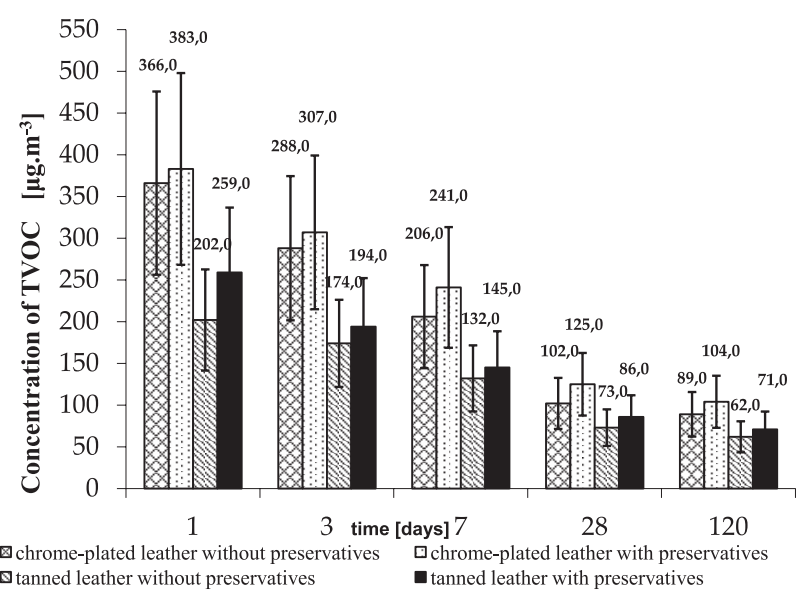

Fig. 3. TVOC from natural leathers based on different type of tanning. 
concentration of 1-Methoxy, 2-propanol was found at measurements after 1 day from chrome-plated leather_ 4 in amount over $36 \mu \mathrm{g} \cdot \mathrm{m}^{-3}$.

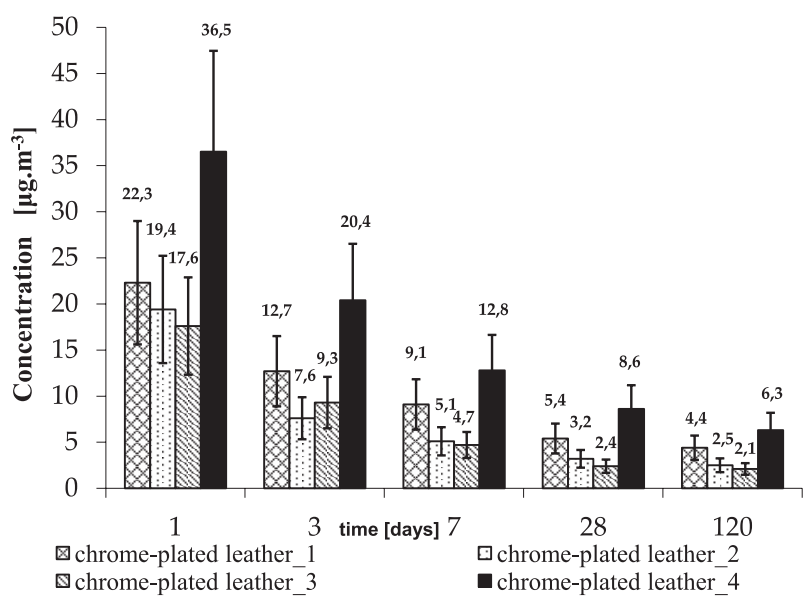

Fig. 4. 1-Methoxy, 2-propanol from different types of upholstery leathers.

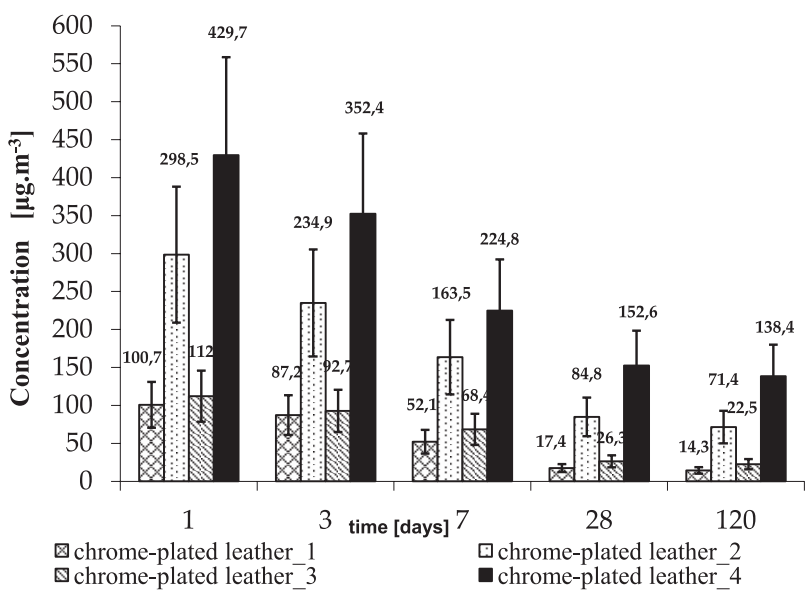

Fig. 5. Butoxy-Ethanol from different types of upholstery leathers.

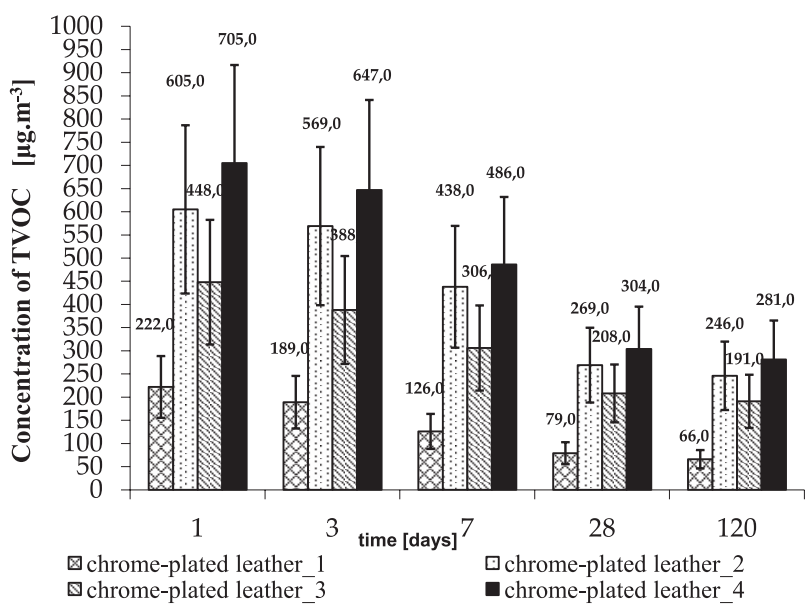

Fig. 6. TVOC from different types of upholstery leathers.
Fig. 5 shows the influence of different types of upholstery leathers on content of emissions VOC, especially emissions of Butoxy-Ethanol. The highest concentration of Butoxy-Ethanol was found at measurements after 1 day from chrome-plated leather no. 4 in amount over $429 \mu \mathrm{g} \cdot \mathrm{m}^{-3}$.

Fig. 6 presents data of parameter TVOC, emitted by different types of upholstery. Parameter of TVOC is describing the total content of Volatile Organic Compounds emitted by selected species of upholstery leathers in depends on time. The highest values of TVOC were measured at measurements after 1 day from leather no. 4 in amount $705 \mu \mathrm{g} . \mathrm{m}^{-3}$, followed by values of concentrations TVOC emitted by chromed leather no. 2 (in amount $605 \mu \mathrm{g} \cdot \mathrm{m}^{-3}$ ).

Based on the results presented in this article, we are able to assess the impact of VOC emissions released from the respective tested materials.

\section{The Influence of the Type of Natural Leather (Animal Origin of Leather) on the Amount of VOC Emissions}

Tables 4-6 show the concentration of VOC emissions from natural upholstery leather in relation to the animal species (buffalo, cow and pig leather). One of the main compounds found in our collection of natural leather VOCs was propylene-glycol-1-methyl ether (synonymum: 1-methoxy-2-propanol, CAS no. 107-98-2). This chemical substance is widely used in primers, highly viscous spray paints and bonding agents in formulated topcoats as constituent additives, or is mainly used in final leather surface finishes. The upholstery leather samples tested released other typical substances such as propylene glycol (synonymum: 1.2 propanediol, CAS no.: 57-55-6); dipropylene glycol monomethyl ether (DPGMME, isomer-1, CAS No.13429-07-7). Propylene glycol is a chemical used mainly in the surface finish process and functions as a coalescence substance for coating applications. Concentrations of other individual volatile organic compounds (VOC) are very low and their values are comparable to background values (from $0.4 \mu \mathrm{g} \cdot \mathrm{m}^{-3}$ for terpenes - to almost $12 \mu \mathrm{g} \cdot \mathrm{m}^{-3}$ for aromatic chemicals, especially toluene). The values of the TVOC (total volatile organic compounds) parameter are not too high (the highest TVOC value was found in natural buffalo hide in quantities above $500 \mu \mathrm{g} . \mathrm{m}^{-3}$ ), but TVOC values decrease depending on the measurement time [24].

\section{The Influence of Different Kinds of Tanning (Chrome-Plated or Tanned leather) on the Amount of VOC Emissions}

The results regarding respective chemicals are shown in Figs 1-2, which demonstrate the influence of different kinds of tanning from the tested materials (natural leather) on the amount of volatile organic 
compounds emitted, especially solvents - namely 1-methoxy, 2-propanol, and butoxyethanol in relation to time, in the course of one month. The measured data show the dependency of the concentration of 1-methoxy, 2-propanol on the type of tested materials. The highest concentration of 1-methoxy, 2-propanol was found when measured after 1 day from chromed leather with preservatives, in an amount greater than $184 \mu \mathrm{g} \cdot \mathrm{m}^{-3}$. But the concentration of butoxyethanol was found only in the amount $28.7 \mu \mathrm{g} . \mathrm{m}^{-3}$ from chromeplated leather with preservatives when measured after 1 day [25].

The data measured reveal the minimal impact of various tested materials on concentrations of BTEX and terpene substances. The concentration of these VOCs was very low, which means that the amount was below the level of quantification (LOQ). The concentration of emitted compounds decreases with time [26].

The values of the so-called total volatile compounds (TVOC) are among the key observed parameters. TVOC represents the total amount of VOCs emitted from the respective materials tested. Fig. 3 shows the influence of the different materials tested on the total amount of VOC emitted in relation to measured time. The highest TVOC values were recorded in a measurement taken after one day from chromium-plated leather with preservatives in the amount of $383 \mu \mathrm{g} . \mathrm{m}^{-3}$, followed by the values of TVOC concentrations emitted by chromeplated leather without preservatives (in the amount of $\left.366 \mu \mathrm{g} . \mathrm{m}^{-3}\right)[27,28]$.

\section{Comparison of VOC Emissions from Different Types of Upholstery Leather}

The chamber test showed differences in the concentration of reactive chemicals from different sample types of natural upholstery leather. The tested samples emitted very high concentrations of butoxyethanol and low amounts of 1-methoxy, 2-propanol. The highest values of VOC emissions were released from natural leather no. 4. The highest concentrations of 1-methoxy, 2-propanol were found only in the amount above $36 \mu \mathrm{g} \cdot \mathrm{m}^{-3}$, whereas the highest concentration value of butoxyethanol was detected in the amount above $629 \mu \mathrm{g} . \mathrm{m}^{-3}$ These test substances were not randomly selected, because 1-methoxy, 2-propanol (Fig. 4) is a typical compound contained in composite coatings as a constitutional compound or is used especially in leather surface finishes [29]. While butoxyethanol (Fig. 5) was found in very high concentrations from the tested samples, the concentrations of BTEX compounds and especially terpene substances were, on the other hand, very low and their values are comparable to the background values (from $0,4 \mu \mathrm{g} \cdot \mathrm{m}^{-3}$ to $4 \mu \mathrm{g} \cdot \mathrm{m}^{-3}$ ) [30].

The results in Fig. 6 show that the trend of releasing TVOC concentrations is in line with the trend of several main volatile substances, such as 1-methoxy, 2-propanol, butoxyethanol, BTEX compounds, and terpenes [31]. All displayed an emission trend in which the concentration decreased with time, eventually reaching a stable level.

\section{Conclusions}

The main objective of this research was to determine the influence of various factors (e.g. the animal species, method of tanning, type of natural leather, etc.) on the amount of VOC emissions used to manufacture upholstered furniture.

The test chamber results showed that the concentrations of VOC emissions from the respective materials tested are impacted not only by the type of natural leather, the animal species or the method of tanning, but also by the different type of the surface treatment used for the leather finishing.

One of the main compounds found in our collection of VOC from natural leather was propylene-glycol1-methyl ether. The upholstery leather test samples released other typical substances, such as propylene glycol and dipropylene glycol monomethyl ether.

\section{Acknowledgements}

The authors are grateful for the support of the Specific University Research Fund of the FFWT Mendel University in Brno of the project „IGA LDF VT_2018002, „The covering materials used for upholstery furniture as a source of emission of VOC and odour in interior "for the financial support.

\section{Conflict of Interest}

The authors declare no conflict of interest.

\section{References}

1. HARB P., LOCOGE N., THEVENET F. Emissions and treatment of VOCs emitted from wood-based construction materials: Impact on indoor air quality. Chemical Engineering Journal, 354, 641, 2018.

2. BARTSCH J., UHDE E., SALTHAMMER T. Analysis of odour compounds from scented consumer products using gas chromatography-mass spectrometry and gas chromatography-olfactometry. Analytica Chimica Acta, 904, 98, 2016.

3. KELLY F. J., FUSSELL J. C. Improving indoor air quality, health and performance within environments where people live, travel, learn and work. Atmospheric Environment, 200, 90, 2019.

4. SALTHAMMER, T. Very volatile organic compounds: an understudied class of indoor air pollutants. Indoor Air, 26 (1), 25, 2016.

5. GONZÁLEZ-MARTÍN J., KRAAKMAN N.J.R., PÉREZ C., LEBRERO R., MUÑOZ R. A state-of-the-art review on 
indoor air pollution and strategies for indoor air pollution control. Chemosphere, 262, 2021.

6. CARON F., GUICHARD R., ROBERT L., VERRIELE M., THEVENET F. Behaviour of individual VOCs in indoor environments: How ventilation affects emission from materials. Atmospheric Environment, 243, 2020.

7. LUCIALLI P., MARINELLO S., POLLINI E., SCARINGI M., SAJANI S. Z., MARCHESI S., CORI L. Indoor and outdoor concentrations of benzene, toluene, ethylbenzene and xylene in some Italian schools evaluation of areas with different air pollution. Atmospheric Pollution Research, 11 (11), 1998, 2020.

8. BRDARIĆ D., KOVAČ-ANDRIĆ E., ŠAPINA M., KRAMARIĆ K., LUTZ N., PERKOVIĆ T., EGOROV A. Indoor air pollution with benzene, formaldehyde, and nitrogen dioxide in schools in Osijek, Croatia. Air Quality, Atmosphere, 12 (8), 963, 2019.

9. CHAPPELL G., POGRIBNY I. P., GUYTON K. Z., RUSYN I. Epigenetic alterations induced by genotoxic occupational and environmental human chemical carcinogens: A systematic literature review. Mutation Research-Reviews in Mutation Research, 768, 27, 2016.

10. KOTZIAS D., KOISTINEN K., KEPHALOPOULOS S., SCHLITT C., CARRER C., MARONI M., JANTUNEN M., COCHET C., KIRCHNER S., LINDVALL T., MCLAUGHLIN J., MØLHAVE L., DE OLIVEIRA FERNANDES E., SEIFERT B., 2005. The INDEX Project: Critical Appraisal of the Setting and Implementation of Indoor Exposure Limits in the EU. Final Report for EUR 21590 EN. Joint Research Center, JRC, Italy. 331 pages.

11. TAGIYEVA N., SEMPLE S., DEVEREUX G., SHEIKH A. Volatile organic compounds and risk of asthma and allergy: a systematic review. European Respiratory Review, 24 (135), 92, 2015.

12. WANG Q., SHEN J., SHAO Y., ET AL., WANG Q., SHEN J., SHAO Y., DONG H., LI Z., SHEN X. Volatile organic compounds and odor emissions from veneered particleboards coated with water-based lacquer detected by gas chromatography-mass spectrometry/olfactometry. Eur. J. Wood Prod. 77, 771, 2019.

13. JIANG L., ZHAO Z., SHEN J., MENG J., DONG H. Evaluation of Effects of VOCs Released by MDF on Indoor Air Quality. Linye Kexue/Scientia Silvae Sinicae, 56 (7), 135, 2020

14. WANG Q., ZENG B., SHEN J., WANG H. Effect of lacquer decoration on VOCs and odor release from $\mathrm{P}$. neurantha (Hemsl.) Gamble. Scientific Reports, 10 (1), 1, 2020.

15. HO J., MUDRABOYINA B., SPENCE-ELDER C., RESENDES R., CUNNINGHAM M.F., JESSOP P.G. Water-borne coatings that share the mechanism of action of oil-based coatings. Green Chemistry, 20 (8), 1899, 2018.

16. XIONG J., CHEN F., SUN L., YU X., ZHAO J., HU Y., WANG Y. Characterization of VOC emissions from composite wood furniture: Parameter determination and simplified model. Building, 161, 106237, 2019.

17. XU B., CHEN X., XIONG J. Air quality inside motor vehicles' cabins: A review. Indoor and Built Environment, 27 (4), 452, 2018.

18. FABER J., BRODZIK K., GOŁDA-KOPEK A., ŁOMANKIEWICZ D. Air pollution in new vehicles as a result of $\mathrm{VOC}$ emissions from interior materials. Polish Journal of Environmental Studies, 22 (6), 1701, 2013.
19. WANG CH., YANG X., GUAN J., GAO K., LI Z. Volatile organic compounds in aircraft cabin: Measurements and correlations between compounds. Building and Environment, 78, 89, 2014.

20. GUAN J., GAO KAI, WANG C., YANG X., LIN C. H., LU C., GAO P. Measurements of volatile organic compounds in aircraft cabins. Part I: Methodology and detected VOC species in 107 commercial flights. Building and Environment, 72, 154, 2014.

21. BRODZIK K., FABER J., ŁOMANKIEWICZ D., GOŁDA-KOPEK A. In-vehicle VOCs composition of unconditioned, newly produced cars. Journal of Environmental Sciences, 26 (5), 1052, 2014.

22. GALLEGO E., ROCA F. J., PERALES J. F., GUARDINO $\mathrm{X}$. Comparative study of the adsorption performance of a multi-sorbent bed (Carbotrap, Carbopack X, Carboxen 569) and a Tenax TA adsorbent tube for the analysis of volatile organic compounds (VOCs). TALANTA -OXFORD THEN AMSTERDAM, 81 (3), 916, 2010.

23. HEGENAUER H., ED., Finishing of Leather-Technical qualification for leather processing professions, 8 th edition, 71, (2001), Heyer, Essen.

24. FRANK G., KOCH S.E., SINGER D., SCHELLE C. Test Chamber-based VOC Quantification Results of Upholstery Leathers Focused on Statistical Frequency Scales for TVOC and Single Compound Concentrations. Journal of the Society of Leather Technologists and Chemists, 100 (5), 225, 2016.

25. WANG J., MA Z., CHEN L., SUN H.J., FAN W.K. Determination of the Emission of Volatile Organic Compounds from Leather Seats in Environmental Test Chamber. Key engineering materials, 768, 31, 2018.

26. XING L., WANG L., ZHANG R. Characteristics and health risk assessment of volatile organic compounds emitted from interior materials in vehicles: a case study from Nanjing, China. Environmental Science and Pollution Research, 25 (15), 14789, 2018.

27. LV M., HUANG W., RONG X., HE JUNZHOU, YANG $\mathrm{X}$. Source apportionment of volatile organic compounds (VOCs) in vehicle cabins diffusing from interior materials. Part I: Measurements of VOCs in new cars in China. Building, 175, 2020.

28. MEČIAROVÁ L., VILČEKOVÁ S., KISELÁK J. Factors Effecting the Total Volatile Organic Compound (TVOC) Concentrations in Slovak Households. International Journal of Environmental Research and Public Health, 14 (12), 1443-1443, 2017.

29. HAN Y., HU J., XIN Z. Facile preparation of high solid content waterborne polyurethane and its application in leather surface finishing. Progress in Organic Coatings, 130, 8, 2019.

30. WANG H., ZHENG J., YANG T., HE Z., ZHANG P., LIU X., ZHANG M., SUN L., YU X., ZHAO J., LIU X., XU B., TONG L., XIONG J. Predicting the emission characteristics of VOCs in a simulated vehicle cabin environment based on small-scale chamber tests: Parameter determination and validation. Environment International, 142, 2020.

31. YANG S., YANG X., LICINA D. Emissions of volatile organic compounds from interior materials of vehicles. Building and Environment, 170, 2020. 